\title{
Medical management versus surgical management in fibroid uterus
}

\author{
Vilas Namdev Kurude, Sneha Shekharreddy Mutyapwar*
}

Department of Obstetrics and Gynecology, Grand Government Medical College, Mumbai, Maharashtra, India

Received: 06 November 2021

Accepted: 12 December 2021

\section{*Correspondence: \\ Dr. Sneha Shekharreddy Mutyapwar, \\ E-mail: reddysneha479@gmail.com}

Copyright: (c) the author(s), publisher and licensee Medip Academy. This is an open-access article distributed under the terms of the Creative Commons Attribution Non-Commercial License, which permits unrestricted non-commercial use, distribution, and reproduction in any medium, provided the original work is properly cited.

\section{ABSTRACT}

Background: Uterine fibroids (leiomyomas) are most common tumours of the uterine smooth muscles, appear to increase in incidence with age during reproductive years, with a peak in incidence between 35 to 40 years. Signs and symptoms of uterine fibroids include heavy or prolonged menstrual bleeding, pain and pregnancy complications. Current management strategies mainly involve surgical interventions, but choice of treatment is guided by patient's age and desire to preserve fertility or avoid 'radical' surgery such as hysterectomy.

Methods: It is the prospective observational study conducted in inpatients of department of OBGY at tertiary care hospital, admitted during the period of June 2019 to May 2021. Outcome measured in terms of relief of symptoms, decrease in size of fibroid, requiring subsequent surgery, quality of life, blood transfusion requirements, length of stay in hospital, successful pregnancies

Results: Of patients treated with ulipristal, $78.57 \%$ had improved quality of life, in patients treated with leuprolide, $57.14 \%$ showed improved quality of life, with mifepristone $80 \%$ patients showed improved quality of life and with LNG $100 \%$ patients responded to it. All patients treated surgically, showed relief of symptoms.

Conclusions: Medical line of management is best for patients in younger age group, small size fibroid, desire for future fertility. Uterus sparing option like myomectomy is done in patients not responding to medical line of management and desire for future fertility. Hysterectomy is definitive line of management for patients with fibroid uterus.

Keywords: Fibroid, Mifepristone, Ulipristal, LNG, Leuprolide, Hysterectomy, Myomectomy

\section{INTRODUCTION}

Uterine fibroids(leiomyomas) are most common tumors of the uterine smooth muscles, appear to increase in incidence with age during reproductive years, with a peak in incidence between 35 to 40 years. $^{1,2}$ They are monoclonal tumors of the smooth muscle cells of the myometrium and consist of large amount of extracellular matrix that contains collagen, fibronectin and proteoglycan. ${ }^{3,4}$

Fibroids occur in $50-60 \%$ of women, rising to $70 \%$ by the age of 50 years and in $30 \%$ of cases cause morbidity due to abnormal uterine bleeding (heavy menstrual bleeding causing anemia) and pelvic pressure (urinary symptoms, constipation and tenesmus). ${ }^{5}$
African- American women are at greater risk of being affected by uterine fibroids. Menarche at an early age increases the risk of developing fibroids, while pregnancy has been found to have a protective effect. Uterine fibroids can be classified into three distinct types, according to their anatomic location-submucosal, intramural, subserosal. ${ }^{6}$

Signs and symptoms of uterine fibroids include heavy or prolonged menstrual bleeding, pain and pregnancy complications. $^{7}$ Leiomyoma diagnosed by pelvic examination, ultrasound, hysteroscopy, computed tomography, laparoscopy and MRI.

Magnetic resonance imaging (MRI) provides information on number of fibroids, their size and location, vascularization, relationship with endometrial cavity and 
serosal surface and boundaries with normal myometrium. ${ }^{6}$ Pharmacological management of fibroid includes oral contraceptives, progestins, progesterone receptor antagonist mifepristone, selective progesterone receptor modulator (SPRM) ulipristal, which has mixed progesterone agonist/antagonist and GnRH agonist and antagonist.

Surgical treatment includes hysterectomy (abdominal, vaginal and laparoscopic) and myomectomy (laparotomy, laparoscopic and hysteroscopic). New approaches, such as myolysis, focused ultrasound, transvaginal cryomyolysis and uterine artery embolization (UAE), laparoscopic uterine artery occlusion (LUAO), doppler guided uterine artery occlusion (D-UAO).

Current management strategies mainly involve surgical interventions, but choice of treatment is guided by patient's age and desire to preserve fertility or avoid 'radical' surgery such as hysterectomy. ${ }^{8}$

\section{METHODS}

It is the prospective observational study conducted in inpatients of department of OBGY at tertiary care hospital, admitted during the period of June 2019 to May 2021. All women diagnosed of fibroid uterus and were admitted in hospital are included in the study. All pregnant women are excluded from the study. Fibroid showing changes of leiomyosarcoma are excluded from study.

\section{Diagnosis}

Fibroid uterus diagnosed by abdominal examination, bimanual examination, with confirmation by ultrasound, MRI, hysteroscopy or laparoscopy.

\section{Management}

Choice of treatment decided by considering age, desire to become pregnant in future, importance of uterine preservation, symptom severity. Medical treatment given for women of younger age, perimenopausal with less severe symptoms, desire for future fertility. GnRH agonists such as leuprolide, SPRMs like ulipristal acetate, progesterone receptor antagonist such as mifepristone, levonorgestrel IUDs (Mirena) are the medical line of management. Injection leuprolide $3.75 \mathrm{mg}$ monthly for 3 months is given. Tablet ulipristal acetate $5 \mathrm{mg}$ daily od for 3 months given. Tablet mifepristone $25 \mathrm{mg}$ OD daily for 3 months. Surgeries include myomectomy and hysterectomy (abdominal, vaginal and laparoscopic).

\section{Outcomes}

Outcome measured in terms of relief of symptoms, decrease in size of fibroid, requiring subsequent surgery, quality of life, blood transfusion requirements, length of stay in hospital, successful pregnancies after medical line of management.
Outcomes of surgical management measured in terms of quality of life, requirement of blood transfusion, length of stay in hospital and successful pregnancies.

\section{RESULTS}

80 women with fibroid uterus admitted at our hospital were included in the study, of which 42 women were given medical line of management and 38 women have undergone surgery. The characteristics such as age, parity, desire for future fertility, size of fibroid is studied. The 10 patients after medical line of management have undergone surgery.

Maximum incidence of uterine fibroid seen in the age group of 35 to 55 years. $58(72 \%)$ patients were in the age group of 35 to 55 years, 19 (24\%) patients were below 35 years and $3(4 \%)$ patients were above 55 years.

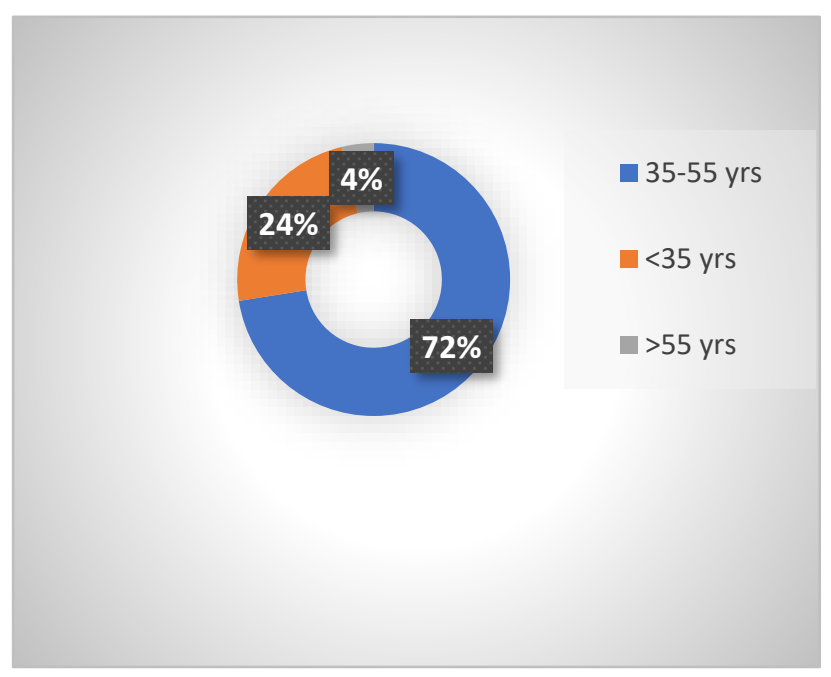

Figure 1: Age incidence of uterine fibroid.

Of 80 women with fibroid uterus, 22 patients $(27.5 \%)$ were nullipara and 13 patients $(16.25 \%)$ were primipara. Both these group of patients desired future fertility and were considered for medical line of management. Remaining patients were multipara $(56.25 \%)$.

Amongst the 80 patients with fibroid uterus, 30 patients $(37.5 \%)$ had fibroid size less than $8 \mathrm{~cm}$ and 50 patients $(62.5 \%)$ had size of more than or equal to $8 \mathrm{~cm}$. Among these 50 patients, 12 patients $(24 \%)$ were given medical line of management and only 2 patients (16.67\%) responded while remaining 10 patients $(83.33 \%)$ landed up in surgery.

Among the 42 patients $(52.5 \%)$ treated with medical line of management, 14 patients $(33.34 \%)$ treated with tablet ulipristal acetate $5 \mathrm{mg}$ od for 3 months. After 3 months, 11 patients $(78.57 \%)$ had relief of symptoms and quality of life improved while, 3 patients $(21.42 \%)$ had no relief of symptoms and required surgery subsequently. Among the 14 patients treated with ulipristal, size of fibroid was decreased in 13 patients $(92.85 \%)$. 


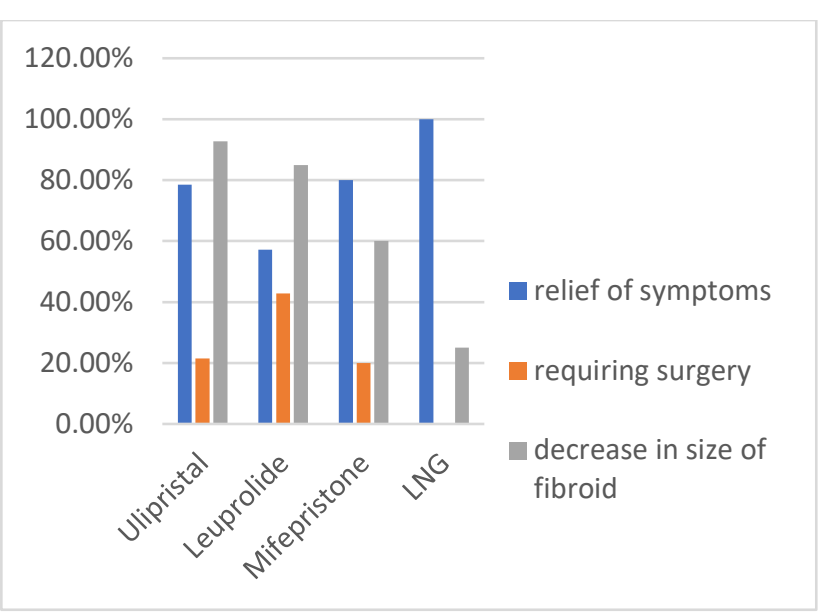

Figure 2: Medical management.

The 14 patients $(33.34 \%)$ were given injection leuprolide $3.7 \mathrm{mg}$ monthly for 3 months, 8 patients $(57.14 \%)$ showed relief of symptoms and improved quality of life, remaining 6 patients $(42.85 \%)$ underwent surgery. The size of fibroid decreased in 12 patients $(85.71 \%)$.

The 10 patients $(23.81 \%)$ treated with tablet mifepristone $25 \mathrm{mg}$ OD daily for 3 months, among these 8 patients $(80 \%)$ showed relief of symptoms and improved quality of life, 2 patients $(20 \%)$ needed subsequent surgery. In 6 patients $(60 \%)$ treated with mifepristone, size of fibroid decreased. In 4 patients (9.5\%), LNG was inserted and all these patients had improved quality of life, and size of fibroid decreased in only 1 patient $(25 \%)$.

Amongst the 38 patients $(47.5 \%)$ treated surgically, in 7 patients $(20 \%)$ myomectomy was done, 2 patients $(6 \%)$ underwent vaginal hysterectomy, in 2 patients $(6 \%)$ laparoscopic assisted vaginal hysterectomy done (LAVH) and 24 patients $(68 \%)$ underwent total abdominal hysterectomy (TAH). All patients treated surgically had relief of symptoms, had improved quality of life.

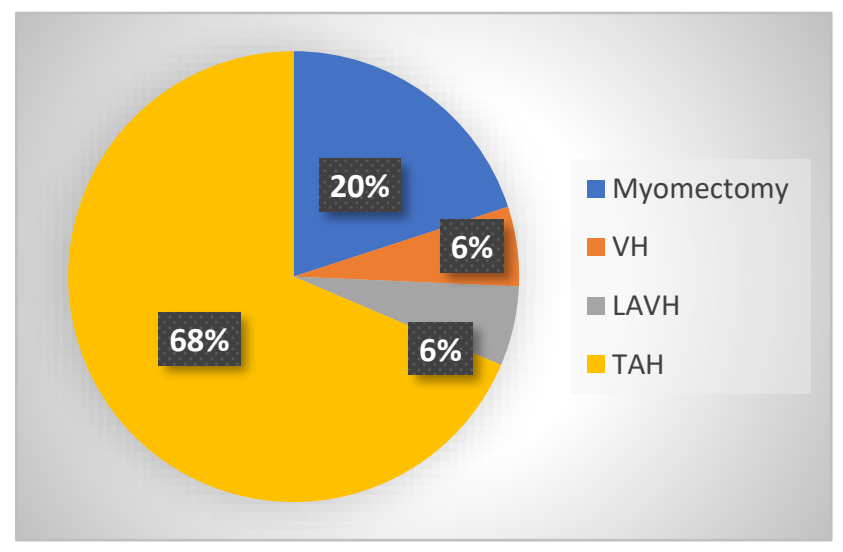

Figure 3: Surgical management.

Among the 7 myomectomies, 5 patients (71.42\%) had successful pregnancies while all 7 desired future pregnancies. Out of 14 patients treated with ulipristal, 6 patients desired future fertility, 4 patients $(66.67 \%)$ had successful pregnancies. Among the 14 patients treated with leuprolide, 5 desired future pregnancy, 3(60\%) had successful pregnancies. Patients treated with mifepristone and LNGs had no desire for future fertility.

Among the 42 patients treated medically, 3 patients needed blood transfusion. In 38 patients treated surgically, 14 patients needed blood transfusion.

Length of stay in hospital is more with surgical line of management. Among the surgeries length of stay is higher in total abdominal hysterectomy and is less in myomectomy patients.

\section{DISCUSSION}

Our study demonstrates the best choice of treatment for patients with primary diagnosis of uterine fibroids, considering the factors such as size of fibroid, age of the patient, desire for future fertility. Patient with small size fibroid (size $<5 \mathrm{~cm}$ ) and/or desire for future fertility should be considered for medical line of management.

For younger patients, medical line of management is best and among the medical line of management, ulipristal and mifepristone have come up with best results, in terms of improvement in lifestyle, relief of symptoms, successful future pregnancies and decrease in size of fibroid.

Ulipristal reduce the volume of leiomyoma through mechanism that include inhibition of leiomyoma cell proliferation, induction of apoptosis, and facilitation of extracellular matrix breakdown and reorganization. ${ }^{9}$ Donnez et al study concluded that ulipristal effectively control bleeding and shrink fibroids in patients with symptomatic fibroids. Levens et al study found ulipristal effective for reducing the size of individual fibroids and the overall fibroid burden as measured by total fibroid and uterine volume. A single course of 5 or $10 \mathrm{mg}$ reduced fibroids size by 17 to $38 \%$.

GnRH agonists are more commonly used for preoperatively decrease the volume of fibroid. Continuous GnRH agonist administration acts to produce hypomenorrhea or amenorrhea and can reduce size of fibroid. ${ }^{10} \mathrm{GnRH}$ agonist are not useful in when fibroids are predominantly calcified. Carbonell et al studies stated that 68 to 100 percent of women in their trials reported pelvic pain. By three months of treatment, this was reduced to a range of 9 to 28 percent with those in lower dose groups having lower/equivalent prevalence of pelvic pain.

LNG primarily used for contraception, but also used in abnormal uterine bleeding-leiomyoma. ${ }^{11,12}$ LNG delivers $20 \mathrm{mcg}$ per day to inner wall of uterus for at least 7 years, which causes continuous, strong suppression of uterine endometrium. ${ }^{13}$ LNG just reduces menorrhagia in fibroid uterus, it has nothing to do with the size of fibroid. Tosun et al suggested that LNG- IUD can improve bleeding even 
among women whose fibroid symptoms were considered appropriate for surgical intervention. However, quality of study was poor thus evidence to guide care is inadequate.

Patients with large size fibroid and/or not responding to medical line of management, wish to conserve uterus, myomectomy should be taken into consideration that don't render the patient sterile. But patients undergoing myomectomy may have risk of persistence of symptoms, fibroid may recur and require further surgery.

Successful pregnancies after myomectomy may have risk of uterine rupture and may affect mode of delivery. Although hysterectomy continues to be most common treatment for symptomatic uterine fibroid, long-term health effect remains poorly understood, and hysterectomy is not option for women wishing to preserve fertility. Fedele et al study compared GnRH agonist to myomectomy/ hysterectomy. Symptom improvement and fertility outcomes reported. 3 of 5 women who underwent myomectomy for infertility had term pregnancies.

Hysterectomy is preferred by the patients with low socioeconomic status as patients need to get treated in one setting, patient cannot afford medical line of management. Surgical morbidity/mortality such as blood loss, bowel injury, bladder/urethral injury, infection, postoperative pain and death have all been reported with hysterectomy. Typical recovery time for hysterectomy are approximately 6-8 weeks. ${ }^{14}$ LAVH is now-a-days preferred surgical line of management in small size fibroid uterus. Hwang et al and Seracchioli et al studies concluded that women who received a vaginal or laparoscopic vaginal hysterectomy had a significantly faster recovery (mean 22 to 30 days) compared with total abdominal hysterectomy (mean 36 to 41 days) as reported in two studies.

\section{CONCLUSION}

The choice of treatment must be individualized to the women's need and her clinical presentation. Medical line of management is best for patients in younger age group, small size fibroid, desire for future fertility. In medical line of management, ulipristal and mifepristone have better outcomes. Uterus sparing option like myomectomy is done in patients not responding to medical line of management and desire for future fertility. GnRH agonists mainly used to reduce size of fibroid pre-op. Hysterectomy is definitive line of management for fibroid uterus. Promising new treatment approaches (ulipristal, mifepristone) being developed with goals of being effective, safe and less invasive than surgical line of management.

Funding: No funding sources Conflict of interest: None declared
Ethical approval: The study was approved by the Institutional Ethics Committee

\section{REFERENCES}

1. Solomon LA, Schimp VL, Ali-Fehmi R, Diamond MP, Munkarah AR. Clinical update of smooth muscle tumors of the uterus. $\mathrm{J}$ minimally invasive gynecol. 2005;12(5):401-8.

2. Guarnaccia MM, Rein MS. Traditional surgical approaches to uterine fibroids: abdominal myomectomy and hysterectomy. Clin obstetr gynecol. 2001;44(2):385-400.

3. Sankaran S, Manyonda IT. Medical management of fibroids. Best Practice Res Clin Obstetr Gynaecol. 2008;22(4):655-76.

4. Parker WH. Etiology, symptomatology, and diagnosis of uterine myomas. Fertility and sterility. 2007;87(4):725-36.

5. Donnez J, Jadoul P. What are the implications of myomas on fertility? A need for a debate? Human reproduct. 2002;17(6):1424-30.

6. Dolmans MM, Donnez J, Fellah L. Uterine fibroid management: Today and tomorrow. J Obstetrics Gynaecol Res. 2019;45(7):1222-9.

7. American College of Obstetricians and Gynecologists. ACOG practice bulletin. Alternatives to hysterectomy in the management of leiomyomas. Obstetr gynecol. 2008;112(2 Pt 1):387-400.

8. Levy BS. Modern management of uterine fibroids. Acta obstetricia et gynecologica Scandinavica. 2008;87(8):812-23.

9. Courtoy GE, Donnez J, Marbaix E, Dolmans MM. In vivo mechanisms of uterine myoma volume reduction with ulipristal acetate treatment. Fertility and sterility. 2015;104(2):426-34.

10. Chillik C, Acosta A. The role of LHRH agonists and antagonists. Reproductive Bio Med Online. 2001;2(2):120-8.

11. Xiao B, Zeng T, Wu S, Sun H, Xiao N. Effect of levonorgestrel-releasing intrauterine device on hormonal profile and menstrual pattern after longterm use. Contraception. 1995;51(6):359-65.

12. Harrison-Woolrych M, Raine JM. Levonorgestrel intrauterine device can be left in place for five years. Bmj. 1998;317(7151):149.

13. Luukkainen T. Levonorgestrel-Releasing Intrauterine Device. Ann New York Academy Sci. 1991;626(1):43-9.

14. Farquhar CM, Harvey SA, Yu Y, Sadler L, Stewart AW. A prospective study of 3 years of outcomes after hysterectomy with and without oophorectomy. Ame j obstetric gynecol. 2006;194(3):711-7.

Cite this article as: Kurude VN, Mutyapwar SS. Medical management versus surgical management in fibroid uterus. Int J Reprod Contracept Obstet Gynecol 2022;11:87-90. 